\title{
Partial compensation/responsibility
}

\author{
Erwin Ooghe*
}

February 19, 2014

\begin{abstract}
Many theories of fairness distinguish between compensation factors ('luck') and responsibility factors ('effort'). Whereas the distinction between both type of factors is a matter of definition in theory, empirical work usually requires a sharp cut. All determinants of the outcome of interest have to be classified as either a compensation factor or a responsibility factor. We argue that the determinants are often hard to classify. A pragmatic solution to the problem at hand is to introduce a more general soft cut: determinants can be partly compensation, partly responsibility. Still, in a first-best income tax framework, such a soft cut is possible only if the gross income function is additively separable. In case separability fits the data, a simple partial sharing rule emerges as a natural candidate for partial redistribution. This rule can be characterized on the basis of two simple properties, equal treatment of equals and partial solidarity. In case additive separability is rejected by the data, we propose two alternative solutions.
\end{abstract}

Keywords: equality of opportunity - compensation - responsibility.

JEL-codes: D6, D7, H2, I3.

*Department of Economics, KULeuven, erwin.ooghe@kuleuven.be. I would like to thank the editor and an anonymous referee for helpful comments. 


\section{Motivation}

The standard way in economics to assess, improve, or optimize public policy is based on welfarism: welfare in society is measured by an increasing function of subjective individual utilities only. There are different reasons why using subjective utilities can be objectionable. Rawls (1971) criticizes the welfarist approach and argues in favour of equalizing an objective index of primary goods. In the aftermath of Rawls' influential work, many alternative theories of distributive justice were developed; see, e.g., Kymlicka (2002, ch. 3) for an overview. Such theories often share a selective-egalitarian viewpoint: equality is still desirable, but only for differences in outcomes that are caused by a selection of morally irrelevant factors.

The pragmatic theory of responsibility-sensitive egalitarianism proposed by Roemer $(1993,1998)$ and the compensation/responsibility framework proposed by Bossert (1995) and Bossert and Fleurbaey (1996) initiated a body of empirical work; see, e.g., Ramos and Van de gaer (2012) and Roemer and Trannoy (2013) for recent overviews. These pragmatic empirical approaches have two steps in common. The outcome of interest is linked to severable observable determinants that are empirically relevant for the outcome under consideration. Afterwards, a 'sharp' cut is made: each of the determinants, including the inavoidable residual in empirical work, is classified as either a compensation or a responsibility factor.

As recognized by many authors, some of the determinants are hard to classify. We provide some examples. First, the residual is the outcome part that cannot be explained by the empirical model and captures, e.g., omitted compensation and responsibility factors. It is therefore by definition difficult to interpret the residual, let alone classify. The residual also turns out to be of considerable size in most applications and, as a consequence, results can heavily depend on how one classifies it. Second, some determinants like

gender can influence outcomes via different channels, e.g., via opportunities and via preferences. Preferences can be considered a legitimate source of 
outcome differences if individuals identify with their preferences. If one only wants to compensate for the effect of gender on opportunities, one must be able to separate both channels, e.g., by estimating a structural model. In the absence of good instruments, such models are plagued by identification problems. Third, other determinants, like educational attainment, are usually considered to be under partial control, say, a combination of intelligence and diligence. A second-best setting in which taxes induce agents to reveal their private information could be one solution, but the resulting multidimensional screening exercise is rather cumbersome. ${ }^{1}$ Fourth, even if we know that certain variables like genetic defects are inborn and therefore beyond control, there could be other considerations, like cost containment, such that full compensation is not desirable or simply not feasible.

A pragmatic way to deal with such problems is to introduce a soft cut: some of the determinants can be partly compensation and partly responsibility. From a normative point of view, a soft cut can enrich some of the existing pragmatic theories of fairness. Of course, it cannot answer the question to what extent we should compensate individuals for the different underlying determinants. There is probably no universal answer to this question, e.g., because the degree of compensation for a determinant could depend on the outcome under consideration. In our view, the soft cut allows the researcher to adopt a range of reasonable values that can tackle some of the problems mentioned before in a pragmatic way.

Also from a descriptive point of view a soft cut can be better suited to capture individual or social preferences for redistribution. Stated and revealed attitudes towards social spending show a stable pattern over countries and time: individuals are most supportive to compensate for age, followed by sickness and disability, less for needy families with children, and less again for unemployment; see Coughlin (1980). Such individual or social preferences

\footnotetext{
${ }^{1}$ Ooghe and Peichl (2014) introduce simplifying assumptions to keep analytical tractability. Their results are in the end similar to the pragmatic proposal in this paper.
} 
cannot be captured by a sharp cut. There is similar evidence in Gaertner and Schokkaert (2012) that a soft cut based on (what these authors call) intermediate compensation is closer to the opinions on distributive justice in different countries.

In section 2, we model gross income as a function of different factors, but in our model these factors can be partitioned into 'compensation' groups, i.e., subsets of factors with the same degree of compensation. The model of Bossert and Fleurbaey (1996) is a special case with two subsets of factors, one set with full compensation and one with no compensation (or full responsibility).

The central axiom of partial solidarity is introduced in section 3 . It requires that the part of a gross income shock for which the individual is responsible, should be borne by that individual only, while the remaining part should be spread equally over all individuals in society.

Section 4 provides the two main results. First, the introduction of a 'soft' cut based on the idea of partial solidarity does not escape the Bossert (1995) and Fleurbaey (1995) separability result: it can be satisfied only if the gross income function is additively separable between the different compensation groups. In case additive separability fits the data, a simple partial sharing rule emerges as a natural candidate for partial redistribution. A second result characterizes this partial sharing rule on the basis of two simple properties, equal treatment of equals and partial solidarity.

Additive separability is not necessarily satisfied in practice. We discuss in section 5 how to proceed if separability is rejected by the data. A final section 6 concludes.

\section{Notation}

Let $\mathbb{I}$ be a finite set of individuals with cardinality $I \geq 4$ and let $\mathbb{J}$ be a finite set of factors with cardinality $J \geq 2$. Each individual $i \in \mathbb{I}$ is fully

described by a type, i.e., a vector $x_{i} \in \mathbb{R}^{J}$. Let $G: \mathbb{R}^{J} \rightarrow \mathbb{R}$ be the gross 
income function, mapping the type of individual $i$ into his or her gross income $G\left(x_{i}\right)$. The different types in society are collected in a type profile $\mathbf{x}=\left(x_{i}\right)_{i \in \mathbb{I}}$ and $\mathbb{D} \equiv \mathbb{R}^{I \times J}$ is the corresponding domain.

The planner wants to redistribute gross incomes in society. A redistribution scheme is a net income scheme $N: \mathbb{D} \rightarrow \mathbb{R}^{I}$ mapping a type profile x into a vector of net incomes $N(\mathbf{x})=\left(N_{i}(\mathbf{x})\right)_{i \in \mathbb{I}}$ such that budget balance holds, i.e., $\sum_{i \in \mathbb{I}} N_{i}(\mathbf{x})=\sum_{i \in \mathbb{I}} G\left(x_{i}\right)$ for all $\mathbf{x} \in \mathbb{D}$. No behaviour is introduced here.

We generalize the model of Bossert and Fleurbaey (1996) to allow for different groups of factors, each with a different degree of compensation. The set of factors $\mathbb{J}$ can be partitioned into $P$ different subsets denoted $\mathbb{J}^{1}, \mathbb{J}^{2}, \ldots, \mathbb{J}^{P}$, such that all factors with the same degree of compensation end up in the same 'compensation' group. We gather these degrees of compensation in a vector $\gamma=\left(\gamma^{1}, \gamma^{2}, \ldots, \gamma^{P}\right) \in \mathbb{R}^{P}$, with $\gamma^{k} \neq \gamma^{\ell}$ if $k \neq \ell$. For ease of exposition, we decompose the type of an individual as $x_{i}=\left(x_{i}^{1}, x_{i}^{2}, \ldots, x_{i}^{P}\right)$,

with $x_{i}^{k}$ the subvector of $x_{i}$ corresponding with the factors in compensation group $k \in \mathbb{P} \equiv\{1,2, \ldots, P\}$. In a compensation group $k$ there can be no compensation $\left(\gamma^{k}=0\right)$, full compensation $\left(\gamma^{k}=1\right)$, and partial compensation $\left(0<\gamma^{k}<1\right)$. In principle, also anti-compensation $\left(\gamma^{k}<0\right)$ and overcompensation $\left(\gamma^{k}>1\right)$ are possible. Opinion research shows for example that many respondents want to anti-compensate in specific cases, probably to deter undesirable behaviours; see, Gaertner and Schokkaert (2012).

\section{Axioms}

We introduce and discuss three axioms: equal treatment of equals, partial solidarity, and partial compensation. Equal treatment of equals requires that two individuals with the same type should receive the same net income. This axiom silently assumes that all relevant characteristics are taken up in the analysis.

Equal treatment of equals. For all $\mathbf{x} \in \mathbb{D}, i, j \in \mathbb{I}$, if $x_{i}=x_{j}$, then 
$N_{i}(\mathbf{x})=N_{j}(\mathbf{x})$

To explain partial solidarity, suppose that the $\ell$-th compensation factor of individual $j$ changes from $x_{j}^{\ell}$ to $x_{j}^{\prime \ell}$, ceteris paribus. This causes a shock in the gross income of individual $j$ equal to $G\left(x_{j}^{\prime}\right)-G\left(x_{j}\right)$. Partial solidarity requires that the part of the gross income shock for which individual $j$ is not (held) responsible, being $\gamma^{\ell}\left(G\left(x_{j}^{\prime}\right)-G\left(x_{j}\right)\right)$, should be borne equally by all individuals (including $j$ ), while the remaining part, $\left(1-\gamma^{\ell}\right)\left(G\left(x_{j}^{\prime}\right)-G\left(x_{j}\right)\right.$ ), should be borne by individual $j$ only.

Partial solidarity. For all $\mathbf{x}, \mathbf{x}^{\prime} \in \mathbb{D}, j \in \mathbb{I}, \ell \in \mathbb{P}$, if $x_{i}=x_{i}^{\prime}$ for each $i \in \mathbb{I} \backslash\{j\}$, and $x_{j}^{k}=x_{j}^{\prime k}$, for each $k \in \mathbb{P} \backslash\{\ell\}$, then

$$
\begin{aligned}
N_{i}\left(\mathbf{x}^{\prime}\right)-N_{i}(\mathbf{x}) & =\gamma^{\ell}\left(G\left(x_{j}^{\prime}\right)-G\left(x_{j}\right)\right) / I, \text { for all } i \in \mathbb{I} \backslash\{j\}, \text { and } \\
N_{j}\left(\mathbf{x}^{\prime}\right)-N_{j}(\mathbf{x}) & =\gamma^{\ell}\left(G\left(x_{j}^{\prime}\right)-G\left(x_{j}\right)\right) / I+\left(1-\gamma^{\ell}\right)\left(G\left(x_{j}^{\prime}\right)-G\left(x_{j}\right)\right) .
\end{aligned}
$$

In case full compensation applies to compensation group $\ell\left(\gamma^{\ell}=1\right)$, partial solidarity implies 'group solidarity'; if no compensation applies $\left(\gamma^{\ell}=0\right)$, then partial solidarity ensures 'individual monotonicity'; see Bossert (1995) for a definition of both axioms.

Partial compensation is similar to partial solidarity, but it is concerned with type differences between individuals rather than type changes of individuals. Suppose that the gross income difference between two individuals can be fully attributed to differences in the factors of one compensation group only. Partial compensation requires that the net income difference between these individuals should be proportional to their gross income difference, and the degree of responsibility-i.e., one minus the degree of compensation - is the proportionality factor.

Partial compensation. For all $\mathbf{x} \in \mathbb{D}, i, j \in \mathbb{I}, \ell \in \mathbb{P}$, if $x_{i}^{k}=x_{j}^{k}$, for each $k \in \mathbb{P} \backslash\{\ell\}$, then $N_{i}(\mathbf{x})-N_{j}(\mathbf{x})=\left(1-\gamma^{\ell}\right)\left(G\left(x_{i}\right)-G\left(x_{j}\right)\right)$.

In case full compensation applies to compensation group $\ell\left(\gamma^{\ell}=1\right)$, partial compensation becomes 'equal income for equal responsibility'; if no compensation applies $\left(\gamma^{\ell}=0\right)$, then partial compensation reduces to 'equal transfer 
for equal skills'; see Bossert and Fleurbaey (1996) for a definition of both axioms.

\section{The partial sharing rule}

Before we provide the partial sharing rule and its characterization, we discuss two interesting consequences of the axioms. First, partial solidarity and equal treatment of equals imply partial compensation.

LEMMA 1. If a redistribution scheme satisfies PARTIAL SOLIDARITY and EQUAL TREATMENT OF EQUALS, then it satisfies PARTIAL COMPENSATION. Second, partial compensation requires the gross income function $G$ to be additively separable over the different compensation groups. We say that the gross income function $G$ is additively separable over the compensation groups in $\mathbb{P}$ if there exist a function $G^{k}: \mathbb{R}^{J^{k}} \rightarrow \mathbb{R}$ for each compensation group $k \in \mathbb{P}$ such that $G(x)=\sum_{k \in \mathbb{P}} G^{k}\left(x^{k}\right)$ for each $x \in \mathbb{R}^{J}{ }^{2}$

LEMMA 2. If a redistribution scheme satisfies PARTIAL COMPENSATION, then the gross income function $G$ must be additively separable over the compensation groups in $\mathbb{P}$.

Lemma 1 and 2 together imply that partial solidarity and equal treatment of equals cannot be combined unless the gross income function is additively separable. Whether separability holds is ultimately an empirical question. The answer will depend on the data and on the chosen partitioning of the set of factors. If additive separability fits the data, the following partial sharing rule generalizes Bossert's (1995, equation 1, p. 2) rule and is a natural candidate for partial redistribution. A redistribution scheme is said to be the partial sharing rule if, for all $\mathbf{x} \in \mathbb{D}$, it assigns a net income

$$
N_{i}(\mathbf{x})=\underbrace{\frac{1}{I} \sum_{i \in \mathbb{I}} \sum_{k \in \mathbb{P}} \gamma^{k} G^{k}\left(x_{i}^{k}\right)}_{(1)}+\underbrace{\sum_{k \in \mathbb{P}}\left(1-\gamma^{k}\right) G^{k}\left(x_{i}^{k}\right)}_{(2)}
$$

\footnotetext{
${ }^{2} J^{k}$ refers to the cardinality of compensation group $\mathbb{J}^{k}$.
} 
to individual $i \in \mathbb{I}$. In part (1) the partial sharing rule equally shares those parts of individuals' gross incomes for which individuals are not deemed responsible, and in part (2) it assigns to each individual the part of gross income for which he is deemed responsible. The next proposition summarizes the discussion.

Proposition.

a. If the gross income function is not additively separable over the compensation groups in $\mathbb{P}$, then there is no redistribution scheme satisfying EQUAL TREATMENT OF EQUALS and PARTIAL SOLIDARITY.

b. If the gross income function is additively separable over the compensation groups in $\mathbb{P}$, then the only redistribution scheme satisfying EQUAL TREATMENT OF EQUALS and PARTIAL SOLIDARITY is the partial sharing rule.

\section{Rules without separability}

How can we proceed if the gross income function is not additively separable, e.g., if it has been rejected by the data? We discuss two ways to weaken the crucial axiom, in our case partial compensation or partial solidarity. The first one is based on a reference type and therefore simply extends the existing egalitarian equivalent and conditional egalitarian rules to the current framework. The second one is a more pragmatic solution that is new to the literature.

First, in line with the existing literature we could use a reference type to derive egalitarian-equivalent and conditional egalitarian rules; see, e.g., Fleurbaey (2008, 2.6-2.7). Let $\widetilde{x} \in \mathbb{R}^{J}$ denote a reference type and impose partial compensation only in those cases in which individual factors are equal to the reference type, except for the compensation group under consideration; formally:

Partial Compensation w.R.T. $\widetilde{x}$. For all $\mathbf{x} \in \mathbb{D}, i, j \in \mathbb{I}, \ell \in \mathbb{P}$, if $x_{i}^{k}=$ $x_{j}^{k}=\widetilde{x}^{k}$, for each $k \in \mathbb{P} \backslash\{\ell\}$, then $N_{i}(\mathbf{x})-N_{j}(\mathbf{x})=\left(1-\gamma^{\ell}\right)\left(G\left(x_{i}\right)-G\left(x_{j}\right)\right)$. 
In the Bossert and Fleurbaey (1996) case with two compensation groups, one with no compensation and one with full compensation, the current version of partial compensation encompasses both their axioms of 'equal income for reference responsibility' and 'equal transfer for reference skill'. Define $\left(\widetilde{x}^{-k} ; x_{i}^{k}\right)$ as the reference vector $\widetilde{x}$ in which $\widetilde{x}^{k}$ is replaced by $x_{i}^{k}$, thus $\left(\widetilde{x}^{-k} ; x_{i}^{k}\right)=\left(\widetilde{x}^{1}, \ldots, \widetilde{x}^{k-1}, x_{i}^{k}, \widetilde{x}^{k+1}, \ldots, \widetilde{x}^{P}\right)$. The redistribution scheme assigning

$N_{i}(\mathbf{x})=\sum_{k \in \mathbb{P}}\left(1-\gamma^{k}\right) G\left(\widetilde{x}^{-k} ; x_{i}^{k}\right)+\frac{1}{I} \sum_{i \in \mathbb{I}}\left(G\left(x_{i}\right)-\sum_{k \in \mathbb{P}}\left(1-\gamma^{k}\right) G\left(\widetilde{x}^{-k} ; x_{i}^{k}\right)\right)$

to each $i \in \mathbb{I}$, generalizes the egalitarian-equivalent solution, while

$$
N_{i}(\mathbf{x})=G\left(x_{i}\right)-\sum_{k \in \mathbb{P}} \gamma^{k} G\left(\widetilde{x}^{-k} ; x_{i}^{k}\right)+\frac{1}{I} \sum_{i \in \mathbb{I}} \sum_{k \in \mathbb{P}} \gamma^{k} G\left(\widetilde{x}^{-k} ; x_{i}^{k}\right)
$$

for each $i \in \mathbb{I}$, generalizes the conditional egalitarian one. ${ }^{3}$ It is easily verified that both solutions satisfy partial compensation w.r.t. $\widetilde{x}$. This axiom, or the (multi-profile) solidarity version, could again form the basis of a full characterization.

Second, an alternative more pragmatic solution is to look to what extent separability is rejected by the data. Suppose for example that only one compensation group, say group $P$, cannot be separated from the other ones. More precisely, suppose there exist functions $G^{1}, G^{2}, \ldots, G^{P-1}$ such that $G(x)=\sum_{k=1}^{P-1} G^{k}\left(x^{k}, x^{P}\right)$ for each $x \in \mathbb{R}^{J}$. In this case, the redistribution scheme assigning

$$
N_{i}(\mathbf{x})=\sum_{k=1}^{P-1}\left(1-\gamma^{k}\right) G^{k}\left(x_{i}^{k}, x_{i}^{P}\right)+\frac{1}{I} \sum_{i \in \mathbb{I}} \sum_{k=1}^{P-1} \gamma^{k} G^{k}\left(x_{i}^{k}, x_{i}^{P}\right),
$$

\footnotetext{
${ }^{3}$ In Bossert and Fleurbaey (1996) there are two compensation groups only, one with no compensation, say group 1 with $\gamma_{1}=0$, and one with full compensation, group 2 with $\gamma_{2}=1$. The first solution reduces to

$$
N_{i}(\mathbf{x})=G\left(x_{i}^{1}, \widetilde{x}^{2}\right)-\frac{1}{I} \sum_{i \in \mathbb{I}}\left(G\left(x_{i}^{1}, \widetilde{x}^{2}\right)-G\left(x_{i}\right)\right), \text { for each } i \in \mathbb{I},
$$
}

while the second one becomes

$$
N_{i}(\mathbf{x})=G\left(x_{i}\right)-G\left(\widetilde{x}^{1}, x_{i}^{2}\right)+\frac{1}{I} \sum_{i \in \mathbb{I}} G\left(\widetilde{x}^{1}, x_{i}^{2}\right), \text { for each } i \in \mathbb{I} .
$$


to each $i \in \mathbb{I}$, satisfies partial compensation for each compensation group $\ell$, with $\ell \neq P$; formally:

Partial Compensation w.R.t. Groups $\ell \neq P$. For all $\mathbf{x} \in \mathbb{D}, i, j \in \mathbb{I}$, $\ell \in \mathbb{P} \backslash\{P\}$,if $x_{i}^{k}=x_{j}^{k}$, for each $k \in \mathbb{P} \backslash\{\ell\}$, then $N_{i}(\mathbf{x})-N_{j}(\mathbf{x})=(1-$ $\left.\gamma^{\ell}\right)\left(G\left(x_{i}\right)-G\left(x_{j}\right)\right)$.

This would provide another pragmatic way out without ressorting to reference types.

\section{Conclusion}

Descriptive and prescriptive theories of fairness have been introduced in political economy models, public policy design and equality of opportunity measurement. Some of the more pragmatic empirical approaches require a sharp cut: all determinants of the outcome of interest have to be classified as either 'luck' (a compensation factor) or 'effort' (a responsibility factor).

We argue that some determinants are hard to classify and propose a more flexible soft cut as a pragmatic solution to the classification problem. The corresponding notion of partial compensation/responsibility cannot escape the typical separability requirement of the gross income function in a firstbest income tax model. If additive separability fits the data, we propose and characterize a partial sharing rule as a natural candidate for partial redistribution. We also hint at different ways in which one can proceed if separability is rejected by the data. The introduction of a soft cut does not escape the separability result, but makes the compensation/responsibility theory more flexible and, hopefully, more attractive for practitioners as well. 


\section{References}

[1] Bossert, W., 1995, Redistribution mechanisms based on individual characteristics, Mathematical Social Sciences 29, 1-17.

[2] Bossert, W., Fleurbaey, M., 1996, Redistribution and compensation, Social Choice and Welfare 13, 343-355.

[3] Coughlin, R., 1980, Ideology, Public Opinions and Welfare Policy: Attitudes toward Taxes and Spending in the Industrial Societies, Institute of International Studies Research Series 42, University of California, Berkeley.

[4] Fleurbaey, M., 1995, The requisites of equal opportunity, in Barnett, W., Moulin, H., Salles, M., Schofield, N., eds., Social Choice, Welfare, and Ethics, Cambridge: Cambridge University Press.

[5] Fleurbaey, M., 2008, Fairness, Responsibility and Welfare, Oxford: Oxford University Press.

[6] Gaertner, W., Schokkaert, E., 2012, Empirical Social Choice, Cambridge: Cambridge University Press.

[7] Kymlicka, W., 2002, Contemporary Political Philosophy, Oxford: Oxford University Press.

[8] Ooghe, E., Peichl, A., 2014, Fair and efficient taxation under partial control, ZEW Discussion Paper 14-002.

[9] Ramos, X., Van de gaer, D., 2012, Empirical approaches to inequality of opportunity: principles, measures, and evidence. IZA Discussion Paper 6672 .

[10] Rawls, J., 1971, A Theory of Justice, Oxford: Oxford University Press.

[11] Roemer, J., 1993, A pragmatic theory of responsibility for the egalitarian planner, Philosophy and Public Affairs 22(2), 146-166. 
[12] Roemer, J., 1998, Equality of Opportunity, Harvard: Harvard University Press.

[13] Roemer, J., Trannoy, A., 2013, Equality of opportunity, Cowles foundation discussion paper 1921. 


\section{Proof of lemma 1}

Consider a redistribution scheme $N: \mathbb{D} \rightarrow \mathbb{R}^{I}: \mathbf{x} \mapsto N(\mathbf{x})$ that satisfies PARTIAL SOLIDARITY and EQUAL TREATMENT OF EQUALS. We must show that also partial Compensation is satisfied. More precisely, for each $\mathbf{x} \in \mathbb{D}$, for each individual $i, j \in \mathbb{I}$ and for each compensation group $\ell \in \mathbb{P}$, if $x_{i}^{k}=x_{j}^{k}$, for each $k \in \mathbb{P} \backslash\{\ell\}$ is true, then $N_{i}(\mathbf{x})-N_{j}(\mathbf{x})=\left(1-\gamma^{\ell}\right)\left(G\left(x_{i}\right)-G\left(x_{j}\right)\right)$ must result by combining PARTIAL SOLIDARITY and EQUAL TREATMENT OF EQUALS.

Construct a profile $\mathbf{x}^{\prime}$ with (1) $x_{l}=x_{l}^{\prime}$ for $l \in \mathbb{I} \backslash\{j\}$, and (2) $x_{j}^{\prime}=x_{i}$. In words, the transition from $\mathbf{x}$ to $\mathbf{x}^{\prime}$ is such that individual $j$ becomes a copy of individual $i$, ceteris paribus. This only requirs a change from $x_{j}^{\ell}$ to $x_{j}^{\prime \ell}=x_{i}^{\ell}$, while $x_{j}^{k}=x_{j}^{k}$, for each $k \in \mathbb{P} \backslash\{\ell\}$. We can apply PARTIAL SOLIDARITy to get

$$
N_{j}\left(\mathbf{x}^{\prime}\right)-N_{j}(\mathbf{x})=N_{i}\left(\mathbf{x}^{\prime}\right)-N_{i}(\mathbf{x})+\left(1-\gamma^{\ell}\right)\left(G\left(x_{j}^{\prime}\right)-G\left(x_{j}\right)\right),
$$

for $i$ and $j$. Now, since $x_{i}^{\prime}=x_{j}^{\prime}$ by construction, EQUAL TREATMENT OF EQUALS in profile $\mathbf{x}^{\prime}$ requires $N_{i}\left(\mathbf{x}^{\prime}\right)=N_{j}\left(\mathbf{x}^{\prime}\right)$. Using $N_{i}\left(\mathbf{x}^{\prime}\right)=N_{j}\left(\mathbf{x}^{\prime}\right)$ and $x_{j}^{\prime}=x_{i}$ in equation (1) leads to

$$
N_{i}(\mathbf{x})-N_{j}(\mathbf{x})=\left(1-\gamma^{\ell}\right)\left(G\left(x_{j}^{\prime}\right)-G\left(x_{j}\right)\right)=\left(1-\gamma^{\ell}\right)\left(G\left(x_{i}\right)-G\left(x_{j}\right)\right),
$$

as required.

\section{Proof of lemma 2}

Consider a redistribution scheme $N$ that satisfies PARTIAL COMPENSATION. We must show that there exist functions $G^{1}, G^{2}, \ldots, G^{P}$, one function for each compensation group in $\mathbb{P}=\{1,2, \ldots, P\}$, such that $G(x)=\sum_{k \in \mathbb{P}} G^{k}\left(x^{k}\right)$ for each $x \in \mathbb{R}^{J}$. In case $P=1$ the separability condition is obvious, so we focus on $J \geq 2$ and $2 \leq P \leq J$ in the sequel. 
Step 1. Recall that $J^{k}$ is the cardinality of $\mathbb{J}^{k}$. For any two compensation groups $k$ and $\ell$, with $\ell>k$, we show that there must exist functions $G_{k \ell}^{-\ell}$ : $\mathbb{R}^{J-J_{\ell}} \rightarrow \mathbb{R}$ and $G_{k \ell}^{-k}: \mathbb{R}^{J-J_{k}} \rightarrow \mathbb{R}$ such that

$G(x)=G_{k \ell}^{-\ell}\left(x^{1}, \ldots, x^{\ell-1}, x^{\ell+1}, \ldots, x^{P}\right)+G_{k \ell}^{-k}\left(x^{1}, \ldots, x^{k-1}, x^{k+1}, \ldots, x^{P}\right)$

for each $x \in \mathbb{R}^{J}$. Consider two compensation groups $k$ and $\ell$ with $\ell>k$ and consider four individuals (1, 2, 3 and 4) with types

$$
\begin{aligned}
x_{1} & =\left(x^{1}, \ldots, x^{k-1}, x^{k}, x^{k+1}, \ldots, x^{\ell-1}, x^{\ell}, x^{\ell+1}, \ldots, x^{P}\right) \equiv x, \\
x_{2} & =\left(x^{1}, \ldots, x^{k-1}, x^{k}, x^{k+1}, \ldots, x^{\ell-1}, b, x^{\ell+1}, \ldots, x^{P}\right), \\
x_{3} & =\left(x^{1}, \ldots, x^{k-1}, a, x^{k+1}, \ldots, x^{\ell-1}, x^{\ell}, x^{\ell+1}, \ldots, x^{P}\right), \\
x_{4} & =\left(x^{1}, \ldots, x^{k-1}, a, x^{k+1}, \ldots, x^{\ell-1}, b, x^{\ell+1}, \ldots, x^{P}\right) .
\end{aligned}
$$

for arbitrary vectors $x \in \mathbb{R}^{J}, a \in \mathbb{R}^{J^{k}}$ and $b \in \mathbb{R}^{J^{\ell}}$. PARTial COMPENSATion requires

$$
\begin{aligned}
& N_{1}(\mathbf{x})-N_{2}(\mathbf{x})=\left(1-\gamma^{\ell}\right)\left(G\left(x_{1}\right)-G\left(x_{2}\right)\right), \\
& N_{3}(\mathbf{x})-N_{4}(\mathbf{x})=\left(1-\gamma^{\ell}\right)\left(G\left(x_{3}\right)-G\left(x_{4}\right)\right), \\
& N_{1}(\mathbf{x})-N_{3}(\mathbf{x})=\left(1-\gamma^{k}\right)\left(G\left(x_{1}\right)-G\left(x_{3}\right)\right), \\
& N_{2}(\mathbf{x})-N_{4}(\mathbf{x})=\left(1-\gamma^{k}\right)\left(G\left(x_{2}\right)-G\left(x_{4}\right)\right) .
\end{aligned}
$$

Subtracting (3) from (2) and (5) from (4), and noting that both differences have to be the same, we get:

$$
\begin{gathered}
\left(1-\gamma^{\ell}\right)\left(G\left(x_{1}\right)-G\left(x_{2}\right)-G\left(x_{3}\right)+G\left(x_{4}\right)\right) \\
\left(1-\gamma^{k}\right)\left(G\left(x_{1}\right)-G\left(x_{3}\right)-G\left(x_{2}\right)+G\left(x_{4}\right)\right) .
\end{gathered}
$$

Given $\gamma_{k} \neq \gamma_{\ell}$ and $G\left(x_{1}\right)-G\left(x_{2}\right)-G\left(x_{3}\right)+G\left(x_{4}\right)=G\left(x_{1}\right)-G\left(x_{3}\right)-$ $G\left(x_{2}\right)+G\left(x_{4}\right)$, this is only possible if $G\left(x_{1}\right)-G\left(x_{2}\right)-G\left(x_{3}\right)+G\left(x_{4}\right)=0$, or (using $x_{1}=x$ )

$$
G(x)=G\left(x_{2}\right)+\left(G\left(x_{3}\right)-G\left(x_{4}\right)\right)
$$


for each vector $x \in \mathbb{R}^{J}, a \in \mathbb{R}^{J^{k}}$ and $b \in \mathbb{R}^{J^{\ell}}$. Arbitrarily fixing $a$ and $b$ we can define

$$
\begin{aligned}
G_{k \ell}^{-\ell}\left(\ldots, x^{\ell-1}, x^{\ell+1}, \ldots\right) & \equiv G\left(x_{2}\right) \\
& \equiv G\left(\ldots, x^{k-1}, x^{k}, x^{k+1}, \ldots, x^{\ell-1}, b, x^{\ell+1}, \ldots\right)
\end{aligned}
$$

and

$$
\begin{aligned}
G_{k \ell}^{-k}\left(\ldots, x^{k-1}, x^{k+1}, \ldots\right) \equiv & G\left(x_{3}\right)-G\left(x_{4}\right) \\
\equiv & G\left(\ldots, x^{k-1}, a, x^{k+1}, \ldots, x^{\ell-1}, x^{\ell}, x^{\ell+1}, \ldots\right)- \\
& G\left(\ldots, x^{k-1}, a, x^{k+1}, \ldots, x^{\ell-1}, b, x^{\ell+1}, \ldots\right),
\end{aligned}
$$

which leads to the desired result.

Step 2. We show that there must exist a list of functions $G^{1}, G^{2}, \ldots, G^{P}$ s.t. $G(x)=\sum_{k \in \mathbb{P}} G^{k}\left(x^{k}\right)$ for each $x \in \mathbb{R}^{J}$.

If $P=2$, the representation follows directly from step 1 . We proceed by induction. Consider $P$ compensation groups, with $2 \leq P<J$, and suppose that the existence of functions $G_{k \ell}^{-\ell}: \mathbb{R}^{J-J_{\ell}} \rightarrow \mathbb{R}$ and $G_{k \ell}^{-k}: \mathbb{R}^{J-J_{k}} \rightarrow \mathbb{R}$ for any two compensation groups $k$ and $\ell$, with $k<\ell \leq P$, such that

$G(x)=G_{k \ell}^{-\ell}\left(x^{1}, \ldots, x^{\ell-1}, x^{\ell+1}, \ldots, x^{P}\right)+G_{k \ell}^{-k}\left(x^{1}, \ldots, x^{k-1}, x^{k+1}, \ldots, x^{P}\right)$

holds for each $x \in \mathbb{R}^{J}$, implies additive separability of $G$ (induction hypothesis). We show next that it also holds for $P+1$ groups. Consider a function with $P+1$ groups. From step 1 we know that, for each two compensation groups $k$ and $\ell$, with $k<\ell \leq P+1$, there exist functions $G_{k \ell}^{-\ell}: \mathbb{R}^{J-J_{\ell}} \rightarrow \mathbb{R}$ and $G_{k \ell}^{-k}: \mathbb{R}^{J-J_{k}} \rightarrow \mathbb{R}$ such that

$G(x)=G_{k \ell}^{-\ell}\left(x^{1}, \ldots, x^{\ell-1}, x^{\ell+1}, \ldots, x^{P+1}\right)+G_{k \ell}^{-k}\left(x^{1}, \ldots, x^{k-1}, x^{k+1}, \ldots, x^{P+1}\right)$

for each $x \in \mathbb{R}^{J}$. Using these conditions for arbitrary $k<\ell \leq P$, and using the induction hypothesis, there must exist functions $\bar{G}^{k}\left(\cdot, x^{P+1}\right)$ for 
$k=1, \ldots, P$, such that

$$
G\left(x^{1}, \ldots, x^{P}, x^{P+1}\right)=\sum_{k=1}^{P} \bar{G}^{k}\left(x^{k}, x^{P+1}\right),
$$

for each $x^{1}, \ldots, x^{P}, x^{P+1}$. Now, consider an arbitrary compensation group $k<P+1$. Step 1 applied to $k$ and $P+1$ gives us a representation

$$
\begin{aligned}
G\left(x^{1}, \ldots, x^{P}, x^{P+1}\right)= & G_{k(P+1)}^{-k}\left(x^{1}, \ldots, x^{k-1}, x^{k+1}, \ldots, x^{P+1}\right) \\
& +G_{k(P+1)}^{-(P+1)}\left(x^{1}, \ldots, x^{P}\right)
\end{aligned}
$$

which can be combined with (6) to obtain

$$
\begin{aligned}
\sum_{k=1}^{P} \bar{G}\left(x^{k}, x^{P+1}\right)= & G_{k(P+1)}^{-k}\left(x^{1}, \ldots, x^{k-1}, x^{k+1}, \ldots, x^{P+1}\right) \\
& +G_{k(P+1)}^{-(P+1)}\left(x^{1}, \ldots, x^{P}\right)
\end{aligned}
$$

or equivalently,

$$
\begin{aligned}
\bar{G}\left(x^{k}, x^{P+1}\right)= & G_{k(P+1)}^{-k}\left(x^{1}, \ldots, x^{k-1}, x^{k+1}, \ldots, x^{P+1}\right) \\
& +G_{k(P+1)}^{-(P+1)}\left(x^{1}, \ldots, x^{P}\right)-\sum_{\ell \neq k} \bar{G}\left(x^{\ell}, x^{P+1}\right),
\end{aligned}
$$

for each $x^{1}, \ldots, x^{P}, x^{P+1}$. Fixing all variables, except $x^{k}$ and $x^{P+1}$, we get a representation of $\bar{G}\left(x^{k}, x^{P+1}\right)$ as

$$
\bar{G}\left(x^{k}, x^{P+1}\right)=G^{k}\left(x^{k}\right)+\widetilde{G}_{k}^{P+1}\left(x^{P+1}\right),
$$

with

$$
\begin{aligned}
G^{k}\left(x^{k}\right) \equiv & G_{k(P+1)}^{-(P+1)}\left(\bar{x}^{1}, \ldots, \bar{x}^{k-1}, x^{k}, \bar{x}^{k+1} \ldots, \bar{x}^{P}\right), \\
\widetilde{G}_{k}^{P+1}\left(x^{P+1}\right) \equiv & G_{k(P+1)}^{-k}\left(\bar{x}^{1}, \ldots, \bar{x}^{k-1}, \bar{x}^{k+1}, \ldots, \bar{x}^{P}, x^{P+1}\right) \\
& -\sum_{\ell \neq k} \bar{G}\left(\bar{x}^{\ell}, x^{P+1}\right) .
\end{aligned}
$$

Since this holds for any compensation group $k<P+1$ we can plug it in in equation (6) to obtain the desired result, i.e., the existence of functions $G^{k}$ for $k=1, \ldots, P+1$ such that

$$
G\left(x^{1}, \ldots, x^{P}, x^{P+1}\right)=\sum_{k=1}^{P+1} G^{k}\left(x^{k}\right),
$$

for each $x^{1}, \ldots, x^{P}, x^{P+1}$, with $G^{P+1}\left(x^{P+1}\right)$ equal to $\sum_{k=1}^{P} \widetilde{G}_{k}^{P+1}\left(x^{P+1}\right)$. 


\section{Proof of the proposition}

The first part of the proposition follows directly from lemma 1 and lemma 2 . We prove the second part, i.e., given separability of $G$, PARTIAL SOLIDARITY and EQUAL TREATMENT OF EQUALS lead to the partial sharing rule.

Suppose by contradiction that the partial sharing rule does not follow, i.e., that there exist $\mathbf{x} \in \mathbb{D}$ and $j \in \mathbb{I}$ such that

$$
N_{j}(\mathbf{x}) \neq \sum_{k \in \mathbb{P}}\left(1-\gamma^{k}\right) G^{k}\left(x_{j}^{k}\right)+\frac{1}{I} \sum_{i \in \mathbb{I}} \sum_{k \in \mathbb{P}} G^{k}\left(x_{i}^{k}\right) .
$$

Without loss of generality, we assume

$$
N_{j}(\mathbf{x})>\sum_{k \in \mathbb{P}}\left(1-\gamma^{k}\right) G^{k}\left(x_{j}^{k}\right)+\frac{1}{I} \sum_{i \in \mathbb{I}} \sum_{k \in \mathbb{P}} G^{k}\left(x_{i}^{k}\right) .
$$

Because the budget must be balanced in a redistribution scheme, there must exist some $m \in \mathbb{I}$ such that

$$
N_{m}(\mathbf{x})<\sum_{k \in \mathbb{P}}\left(1-\gamma^{k}\right) G^{k}\left(x_{m}^{k}\right)+\frac{1}{I} \sum_{i \in \mathbb{I}} \sum_{k \in \mathbb{P}} G^{k}\left(x_{i}^{k}\right) .
$$

Both inequalities together imply

$$
N_{j}(\mathbf{x})-N_{m}(\mathbf{x})>\sum_{k \in \mathbb{P}}\left(1-\gamma^{k}\right)\left(G^{k}\left(x_{j}^{k}\right)-G^{k}\left(x_{m}^{k}\right)\right) .
$$

Define $\mathbf{x}(1) \in \mathbb{D}$ to be such that for each $i \in \mathbb{I} \backslash\{j\}, x(1)_{i}=x_{i}$ and for each

$k \in \mathbb{P} \backslash\{1\}, x(1)_{j}^{k}=x_{j}^{k}$ and $x(1)_{j}^{1}=x_{m}^{1}$. Using PARTIAL SOLIDARITy, we get

$$
\begin{aligned}
N_{j}(\mathbf{x}(1))-N_{m}(\mathbf{x}(1)) & =N_{j}(\mathbf{x})-N_{m}(\mathbf{x})+\left(1-\gamma^{1}\right)\left(G\left(x(1)_{j}\right)-G\left(x_{j}\right)\right) \\
& =N_{j}(\mathbf{x})-N_{m}(\mathbf{x})+\left(1-\gamma^{1}\right)\left(G^{1}\left(x_{m}^{1}\right)-G^{1}\left(x_{j}^{1}\right)\right),
\end{aligned}
$$

where the last step follows using additive separability and the construction of $\mathbf{x}(1)$. Combining with (7), we get

$$
\begin{aligned}
N_{j}(\mathbf{x}(1))-N_{m}(\mathbf{x}(1))> & \sum_{k \in \mathbb{P}}\left(1-\gamma^{k}\right)\left(G^{k}\left(x_{j}^{k}\right)-G^{k}\left(x_{m}^{k}\right)\right) \\
& +\left(1-\gamma^{1}\right)\left(G^{1}\left(x_{m}^{1}\right)-G^{1}\left(x_{j}^{1}\right)\right) .
\end{aligned}
$$


Now define $\mathbf{x}(2) \in \mathbb{D}$ to be such that for each $i \in \mathbb{I} \backslash\{j\}, x(2)_{i}=x(1)_{i}$ and for each $k \in \mathbb{P} \backslash\{2\}, x(2)_{j}^{k}=x(1)_{j}^{k}$ and $x(2)_{j}^{2}=x(1)_{m}^{2}$. Note that by construction $x(2)_{j}^{2}=x_{m}^{2}$ and $x(1)_{j}^{2}=x_{j}^{2}$. Applying the same reasoning as above, using equation (8) this time, yields

$$
\begin{aligned}
N_{j}(\mathbf{x}(2))-N_{m}(\mathbf{x}(2))> & \sum_{k \in \mathbb{P}}\left(1-\gamma^{k}\right)\left(G^{k}\left(x_{j}^{k}\right)-G^{k}\left(x_{m}^{k}\right)\right) \\
& +\sum_{k=1}^{2}\left(1-\gamma^{k}\right)\left(G^{k}\left(x_{m}^{k}\right)-G^{k}\left(x_{j}^{k}\right)\right) .
\end{aligned}
$$

Proceeding in this way we end up with a distribution $\mathbf{x}(P)$ such that

$$
\begin{aligned}
N_{j}(\mathbf{x}(P))-N_{m}(\mathbf{x}(P))> & \sum_{k \in \mathbb{P}}\left(1-\gamma^{k}\right)\left(G^{k}\left(x_{j}^{k}\right)-G^{k}\left(x_{m}^{k}\right)\right) \\
& +\sum_{k=1}^{P}\left(1-\gamma^{k}\right)\left(G^{k}\left(x_{m}^{k}\right)-G^{k}\left(x_{j}^{k}\right)\right) .
\end{aligned}
$$

Note that the right-hand side sums up to zero and that $x(P)_{j}=x(P)_{m}$ holds by construction. The inequality $N_{j}(\mathbf{x}(P))>N_{m}(\mathbf{x}(P))$ therefore violates EQUAL TREATMENT OF EQUALS, a contradiction. 